\title{
USO DE ALGORITMOS GENÉTICOS PARA LA CALIBRACIÓN DE UN MODELO HIDROLÓGICO PRECIPITACIÓN- ESCORRENTIA EN LA CUENCA DEL CAPLINA
}

\author{
USING GENETIC ALGORITHMS FOR THE CALIBRATION OF \\ HYDROLOGICAL RAINFALL-RUNOFF MODEL IN THE CAPLINA BASIN
}

${ }^{1}$ Edwin Pino Vargas $;{ }^{2}$ Luis Cornejo Navarretty; ${ }^{3}$ Carmen Román Arce

\section{RESUMEN}

Ante la escasa familiaridad de la ingeniería con los algoritmos genéticos, ¿Cómo estimular su implementación? ¿Cómo mostrar ante los ingenieros las grandes ventajas que se están aprovechando en otros países? Goldberg (1989) dio origen a la teoría formal y al empleo de los algoritmos genéticos. Él dice que se puede adaptar a los campos de la economía, la investigación de operaciones, la medicina, la inteligencia artificial y las ingenierías. El presente trabajo desarrolla metodología y procedimientos, para el uso de los Algoritmos Genéticos, los cuales son una herramienta computacional que nos lleva a la resolución de problemas más complejos a los permitidos por metodologías tradicionales. En nuestro país, los profesionales y los investigadores no están familiarizados con esta técnica. El presente trabajo tiene por objeto calibrar un modelo hidrológico lluvia-escorrentía aplicada a la cuenca Caplina en la región Tacna. Esta cuenca se calibró usando un programa computacional para algoritmos genéticos desarrollado en lenguaje de programación científica MATLAB. Se ha mostrado la evolución del proceso de ajuste de los caudales simulados y observados. En la simulación 1 se aprecia gran diferencia entre estos valores mas no en la simulación 1000, donde se logra un ajuste muy bueno en los meses de estiaje, mas no en los meses de máximas descargas. Las soluciones obtenidas mostraron ser coherentes en similitud a la distribución de caudales observados, con un coeficiente de correlación de 0,7835 .

Palabras clave: algoritmos genéticos, hidroinformática, sistemas inteligentes, inteligencia artificial, soft computing, inteligencia computacional, algoritmos evolutivos, calibración, modelos hidrológicos, modelos lluvia-escorrentía, MATLAB.

\section{ABSTRACT}

Before the unfamiliarity of the engineering in genetic algorithms, how then encourage their implementation? How to display before the engineers the great advantages that if you are taking advantage of it in other countries? According to Goldberg (1989) gave origin to the formal theory and the use of genetic algorithms, he tells us that this can be adapted to the fields such as economics, operations research, medicine, artificial intelligence and engineering, benefited from this new methodology. This paper develops methodology and procedures for the use of genetic algorithms, which in different science and engineering, is a computational tool that takes us to solving more complex than those permitted by traditional methodologies problems. In our country the practitioners and researchers are not familiar with this technique. This paper concerns to calibrate a Hydrological Rainfall-Runoff Model applied to Caplina basin in Tacna region. This basin was successfully calibrated using a computer program for genetic algorithms developed in MATLAB scientific programming language. $n$ this paper we have shown the evolution of the adjustment process of the simulated and observed flows well in simulation 1 shows the large difference between these values in the simulation but not 1000 where a very good fit is achieved in the months of drought, but not in the months of peak discharges. The solutions obtained showed to be consistent in similarity to the distribution of flows observed, with a correlation coefficient of 0,7835 .

Keywords: Genetic algorithms, hydroinformatics, intelligent systems, artificial intelligence, soft computing, computational intelligence, evolutionary algorithms, calibration, hydrological models, models rainfall-runoff, MATLAB.

\section{INTRODUCCIÓN}

Según De Jong (1999), a inicios de los sesentas, las computadoras se volvían cada vez más asequibles y menos costosas. El potencial de estas máquinas fue la atracción de muchos investigadores en Estados Unidos.

John H. Holland (1992), además de su interés por las computadoras, se concentró en otra materia, especialmente, en el estudio de los mecanismos observados en la naturaleza concerniente a la adaptación de las especies. Aplicó dichos mecanismos en la creación de sistemas artificiales (sistemas simulados por computadora).

Fue así como surgió una metodología denominada los algoritmos genéticos. Dichos algoritmos se pueden considerar, entonces, como la adaptación de teorías evolutivas a procedimientos artificiales para la resolución de

' PH.D en Recursos Hídricos, Master of Science en Ingeniería de Recursos Hídricos, Ingeniero Agrícola, Ingeniero Civil. Facultad de Ingenieria Civil, Arquitectura y Geotecnia de la Universidad Nacional Jorge Basadre Grohmann. Tacna-Perú.

${ }^{2}$ Ingeniero Civil. Facultad de Ingenieria Civil, Arquitectura y Geotecnia de la Universidad Nacional Jorge Basadre Grohmann. Tacna-Perú.

${ }^{3}$ Ingeniero Geólogo-Geotecnico. Facultad de Ingeniería Civil, Arquitectura y Geotecnia de la Universidad Nacional Jorge Basadre Grohmann. Tacna-Perú. 
Pino, E. et al., Uso de algoritmos genéticos para la calibración de un modelo hidrológico precipitación-escorrentia en la cuenca del Caplina

problemas. Es por esta razón que en los algoritmos genéticos (o AGs) se encuentran vocablos como cromosomås, poblaciones o mutaciones.

Goldberg (1989), años después con su tesis doctoral, Adaptation in Natural and Artificial Systems (1975) y con la participación de sus estudiantes en la Universidad de Michigan, dio origen a la teoría formal y al empleo de los algoritmos genéticos.

Pronto otros campos como la economía, la investigación de operaciones, la medicina, la inteligencia artifjcial y las ingenierías, se vieron beneficiados por esta nueva metodología.

A pesar de todas las aplicaciones reales que se están dando en el mundo, en el Perú su uso es aún incipiente. No existe material de referencia básico sobre el tema, en todo caso, enfocado a la ingeniería de recursos hídricos, geotecnia y otras ingenierías. Es por esta razón que se realiza el presente trabajo, busca a la vez, incentivar posteriores investigaciones de ingeniería en nuestro país. El presente trabajo brinda no sólo un marco teórico adecuado, sino un software ilustrativo para el estudio de un caso en la cuenca Caplina de la región Tacna relacionado con la hidroinformática, materia especializada en la ingeniería de recursos hídricos.

\section{MATERIALES Y MÉTODOS}

\section{Recolección y análisis de la información hidrometeorológica}

Para la presente evaluación se ha recopilado toda la información pluviométrica existente en el área de estudio, así como, la información cartográfica y otros.

Asimismo, se ha efectuado una revisión de los estudios anteriores. El sistema de medición de variables climáticas, conformado por estaciones hidrometeorológicas de diversos tipos, viene siendo inspeccionada en el campo en forma mensual, con el fin de lograr una buena calidad de datos registrados por parte del Proyecto Especial Tacna y el SENAMHI.

La información existente ha sido analizada estadísticamente utilizando pruebas estándar adecuadas, esto con la finalidad de evaluar su homogeneidad o consistencia.

La región Tacna está compuesta hidrográficamente por un conjunto de cuencas que en su mayoría discurren hacia la vertiente del Pacifico salvo las cuencas Maure y Uchusuma Alto hacia la vertiente el Lago Titicaca.

\section{Modelo hidrológico de Thomas}

Uno de los modelos lluvia-escorrentía que posee la hidrología es el modelo de Thomas. Éste es precisamente el que se empleó para la resolución del caso estudio. En él se definen cuatro parámetros denominados a, b, c, d y dos valores de condición inicial denominados Sw0 y Sg0. Dicho modelo se presenta en la Figura 1.

Los parámetros del modelo de Thomas reflejan las siguientes características de la cuenca:

a: Tendencia de que ocurra escorrentía antes de que el suelo se encuentre completamente saturado.

b: Límite superior de la suma de la evapotranspiración y el

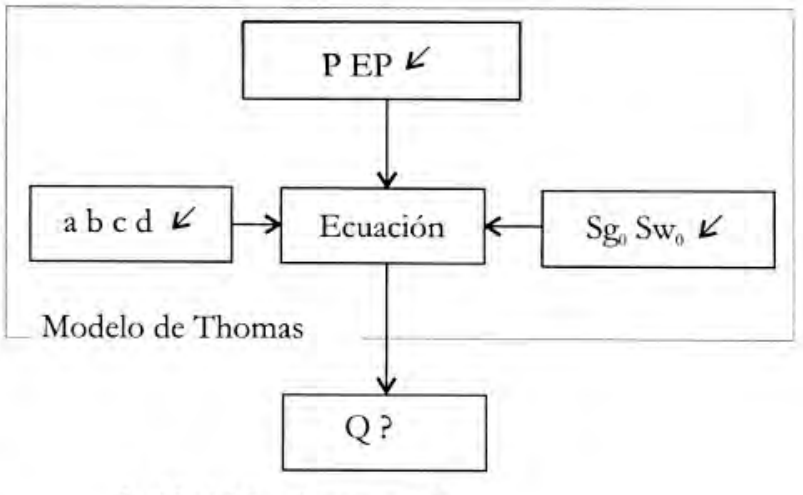

? Variables desconocidas

$\swarrow$ Variables conocidas

Figura 1. Variables en la calibración del modelo de Thomas

contenido de humedad del suelo.

c: Fracción de escorrentía proveniente del agua subterránea.

d: Valor recíproco del tiempo de residencia del agua subterránea.

Las condiciones iniciales hacen referencia a:

$\mathrm{S}_{\mathrm{w}_{0}}$ : Contenido inicial de humedad en el suelo

$\mathrm{Sg}_{0}$ : Almacenamiento inicial de agua subterránea

La ecuación que permite calcular (para un periodo específico) el caudal, a partir de la precipitación P, la evapotranspiración ET y las variables a, b, c, d, Sw $\mathrm{S} \mathrm{Sg}_{\circ}$ es:

$$
\begin{aligned}
& Q=\left\{(1-c)^{*}\left[P+S w_{0}-\frac{P+S w_{0}+b}{2 a}\right]+\left(\frac{P+S w_{0}+b}{2 a}\right)^{2}-\frac{P+S w_{0} * b}{a}\right\}+ \\
& \frac{d}{d+1}\left\{c * \left[P+S w_{0}-\frac{P+S w_{0}+b}{2 a}+\sqrt{\left.\left.\left(\frac{P+S w_{0}+b}{2 a}\right)^{2}-\frac{P+S w_{0}^{*} b}{a}\right]+S g_{0}\right\}}\right.\right.
\end{aligned}
$$

No obstante, dicho cálculo se suele discriminar en una serie de pequeñas ecuaciones que van arrojando, además, otros datos aparte de los caudales. Se sigue entonces la siguiente secuencia hasta calcular finalmente el caudal.

1. Cálculo del agua disponible: $\mathrm{W}=\mathrm{P}+\mathrm{Sw}_{0}$

2. Cálculo de la variable $Y$

$$
Y=\frac{W+b}{2 a} \sqrt{\left(\frac{W+b}{2 a}\right)^{2}-\frac{W^{*} b}{a}}
$$

3. Cálculo del contenido de humedad del suelo: $\mathrm{Sw}=\mathrm{Y}^{*} \mathrm{e}^{-\mathrm{EP} / \mathrm{t}}$

4. Cálculo de la escorrentía directa: $\mathrm{R}_{0}=(1-\mathrm{c}) *(\mathrm{~W}-\mathrm{Y})$

5. Cálculo de la recarga de agua subterránea: $R_{\mathrm{g}}=(\mathrm{c}) *(\mathrm{~W}-\mathrm{Y})$

6. Cálculo del almacenamiento de agua subterránea $S g=\frac{R g+S g_{0}}{d+1}$

7. Cálculo del caudal subterráneo: $\mathrm{Qg}=\mathrm{d} * \mathrm{Sg}$

8. Cálculo del caudal a la salida de la cuenca: $\mathrm{Q}=\mathrm{R}_{0}{ }^{*} \mathrm{Qg}$

Para alcanzar con éxito la habilidad de predecir 
Pino, E. et al., Uso de algoritmos genéticos para la calibración de un modelo hidrológico precipitación-escorrentia en la cuenca del Caplina

caudales se requiere enfrentar dos problemas:

(1) Seleccionar el tipo de modelo que mejor represente el funcionamiento real de la cuenca que se va a analizar.

(2) Determinar los parámetros y condiciones iniciales reales de la cuenca.

Los resultados que arroje el modelo sólo podrán ser confiables en la medida en que los dos problemas se logren superar adecuadamente. En tal sentido, si asumimos que se logró escoger con éxito el modelo (sea por el buen conocimiento de personas expertas, sea por el buen desempeño que ha presentado dicho tipo modelo, etc.) se debe garantizar el correcto conocimiento de los parámetros y las condiciones iniciales. La determinación de los parámetros de proceso presentan el problema de no poderse medir directamente en campo. Aunque los parámetros físicos miden en campo, requieren de ajustes, debido a la posible inexactitud de dichas medidas. La Figura 1 muestra gráficamente cuáles son las variables que se deben conocer para enfrentar el problema de "predecir caudales". Compárese dichas variables con las indicadas en la Figura 2, cuando se trata de enfrentar el problema "calibrar el modelo".

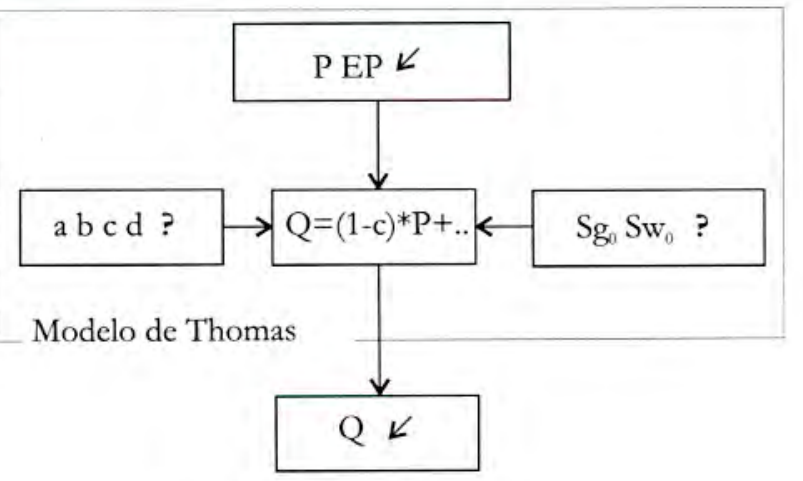

? Variables desconocidas

$\measuredangle$ Variables conocidas

Figura 2. Variables en el problema de la calibración del modelo de Thomas

Para lograr una buena calibración, no se trabaja con un solo valor de P, de EP y de Q en un período de tiempo específico. Se trabaja con varios valores correspondientes a varios períodos de tiempo. En realidad la calibración se logra al reducir a cero algunas de las siguientes funciones (llámense funciones objetivo):

$$
\begin{aligned}
& F(\theta)=\frac{1}{n} \sum_{i=1}^{n}\left[Q o b s_{i}-Q \operatorname{sim}_{i}(\theta)\right]^{\mathrm{P}} \\
& F(\theta)=\frac{1}{n} \sum_{t=1}^{n}\left[Q o b s_{i}-Q \operatorname{sim}_{i}(\theta)\right] \\
& F(\theta)=\max Q o b s_{i}-Q \operatorname{sim}_{i}(\theta)
\end{aligned}
$$

Donde:

Qobs $_{i}=$ caudal medido (obtenido de los archivos)

Q $\operatorname{sim}_{i}(\theta)=$ caudal calculado por el modelo

$\theta=$ conjunto (vector) de los parámetros y condiciones iniciales empleados por el modelo

$\mathrm{n}=$ número de caudales
Finalmente, podemos resumir que el problema de la calibración del modelo de Thomas consiste en encontrar los valores de a, b, c, d, $\mathrm{Sw}_{0}$ y $\mathrm{Sg}_{0}$, de tal manera que la función objetivo escogida sea igual a cero. Para la presente tesis se escogió como función objetivo la denominada norma cuadrática normalizada.

$$
F(\theta)=\frac{1}{n} \sum_{i=1}^{n}\left[Q o b s_{i}-Q \operatorname{sim}_{i}(\theta)\right]^{2}
$$

\section{RESULTADOS Y DISCUSIÓN}

\section{Desarrollo del modelo}

La función objetivo a optimizar es:

$$
F O=\sqrt{n} \sum_{m e s=1}^{12}\left[Q o b s \text { en el } \text { mes }_{i}-Q s i m \text { para el mes }\right]^{2}
$$

Donde $Q_{\text {obs }}$ en el mes está determinado por la ecuación FO. Esto involucra entonces un proceso iterativo. Por consiguiente, es pertinente precisar cuál será la función objetivo a minimizar. En la Figura 3, se muestra el diagrama de flujo para la función objetivo a minimizar.

El cálculo de la función objetivo comienza por obtener externamente a, b, c, d, Sw0 y Sg0. La función tiene dentro de sí, los valores de las precipitaciones, las evaporaciones y los caudales. En amarillo se indica los pasos para calcular cada caudal (y de paso, cada Sw y cada Sg).

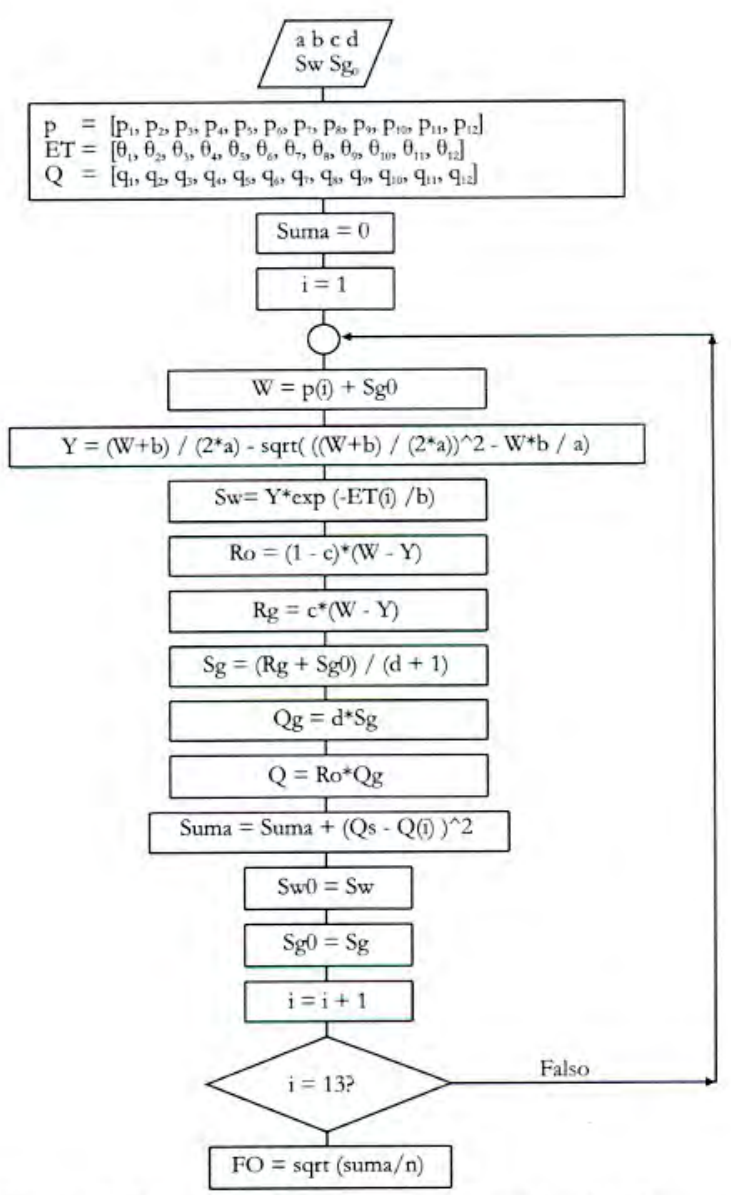

Figura 3. Diagrama de flujo para la función objetivo 
Pino, E. et al., Uso de algoritmos genéticos para la calibración de un modelo hidrológico precipitación-escorrentia en la cuenca del Caplina

\section{Proceso de calibración}

Para el proceso de calibración es fundamental definir el espacio de búsqueda y su respectiva codificación. Trabajos anteriores como el de Karr y Freeman (1999), sugieren rangos entre los cuales pueden variar los parámetros. Ampliando dichos rangos, se determinó que los espacios de búsqueda para cada variable (y las precisiones requeridas) son los indicados en la Tabla 1.

Tabla 1. Rangos de búsqueda y precisión para cada uno de los parámetros

\begin{tabular}{llll}
\hline Parámetro & Mínimo & Máximo & Precisión \\
\hline $\mathrm{a}$ & 0,80 & 1,00 & 0,001 \\
$\mathrm{~b}$ & 10,00 & 350,00 & 1,00 \\
$\mathrm{c}$ & 0,001 & 0,90 & 0,001 \\
$\mathrm{~d}$ & 0,001 & 1,00 & 0,001 \\
$\mathrm{Sw}_{0}$ & 0,00 & 500,00 & 1,00 \\
$\mathrm{Sg}_{0}$ & 0,00 & 500,00 & 1,00 \\
\hline
\end{tabular}

Una vez definido el espacio de búsqueda, se procede a diseñar los cromosomas. La longitud de cada sub-cadena dentro del cromosoma se determina mediante la ecuación:

$$
L=\frac{\ln \left(\frac{W_{\max }-W_{\min }-1}{\pi}\right)}{\ln (k)}
$$

Considerando $\mathrm{k}$ igual 2 para un código binario, las longitudes resultantes son las siguientes:

Para a:

$$
L=\frac{\ln \left(\begin{array}{c}
1,000-0,800 \\
0,001
\end{array}\right)}{\ln (2)} \approx 8
$$

Para b:

$$
L=\frac{\ln \left(\begin{array}{c}
350-1 \\
10^{-1}
\end{array}\right)}{\ln (2)} \approx 9
$$

Para c:

$$
L=\frac{\ln \left(\begin{array}{c}
0,900-0,001 \\
0,001
\end{array}\right)}{\ln (2)} \approx 10
$$

Para d:

$$
L=\frac{\ln \left(\frac{1,000-0,001}{0,001}-1\right)}{\ln (2)} \approx 10
$$

Para $\mathrm{Sw}_{0}$

$$
L=\frac{\ln \left(\begin{array}{c}
500-0 \\
1
\end{array}\right)}{\ln (2)} \approx 9
$$

Para $\mathrm{Sg}$

$$
L=\frac{\ln \left(\begin{array}{c}
500-0 \\
1
\end{array}\right)}{\ln (2)} \approx 9
$$

Figura 4 .
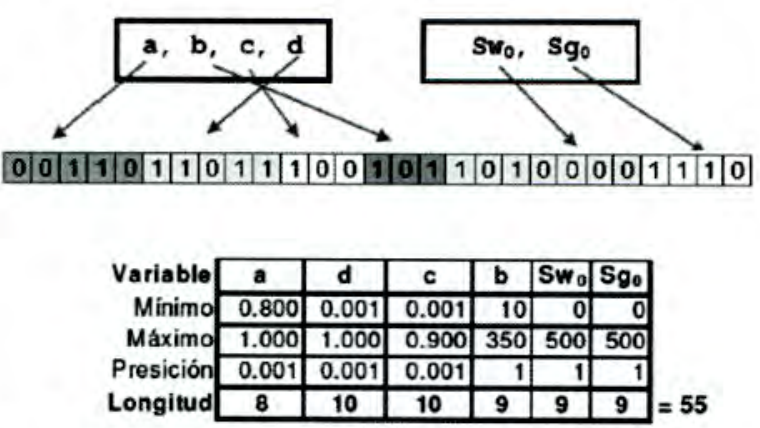

Figura 4. Conformación del tipo de cromosoma a usarse

Si cada cromosoma está compuesto por 55 genes, tal como se indica en la Figura 4, entonces quiere decir que el espacio de búsqueda es de $255=3,6 \times 1016$ posibles soluciones. Esto constituye una razón más por la que se empleó la metodología de los AGs, en el entendido que el espacio de busqueda es muy grande.

El programa elaborado fue desarrollado y se diseñó en lenguaje de programación científica MATLAB R12. La programación tuvo un enfoque estructural (y no orientado a objetos) lo que permite exponer todo su código de manera secuencial. El programa, además de arrojar al final una solución que lograse la minimización de la función objetivo, registra en archivos los resultados de las diferentes generaciones, así como estadísticas que permiten verificar todo el proceso.

El AG finaliza cuando se haya llegado al número de generaciones indicado en MAXGEN o cuando todos los individuos en una generación sean iguales (es decir, cuando se converge a una sola solución).

Se ejecutó el programa cinco veces. Esto es con el fin de ensayar diferentes semillas. Los parámetros ingresados fueron los siguientes:

- $\mathrm{TPOB}=50$

- $\mathrm{MAXGEN}=1000$

- $\mathrm{PX}=0,8$

- $\quad$ PMUT $=0,01$

- $\mathrm{LCAD}=[89101099]$

- MAXVAL = [13500,91500500]

- MINVAL $=[0,8100,0010,00100]$

Cada vez que el programa era ejecutado, se creaba un archivo para cada generación, es decir 1000 archivos. Esta creación de archivos reduce notoriamente la velocidad de ejecución, por tanto simplemente podemos desactivar la subrutina reporte.

\section{Parámetros obtenidos y simulación}

En el Tabla 2, se muestra los resultados de los parámetros obtenidos para el Modelo hidrológico de Thomas, para a, b, c, d Swo y Sgo. Asimismo los caudales calculados por dicho modelo usando los parámetros obtenidos. En la Figura 5 se muestra la comparación de caudales simulados y registrados. La función objetivo que corresponde al cálculo del error en este caso sale de todo rango aceptable y es lógico al observar la gran discrepancia que hay entre los caudales simulados y los registrados a la salida de la cuenca. Retomando la concepción.de la 
Pino, E. et al., Uso de algoritmos genéticos para la calibración de un modelo hidrológico precipitación-escorrentia en la cuenca del Caplina

Tabla 2. Parámetros y simulación usando Generación 1

\begin{tabular}{lccccc}
\hline Mes & $\mathbf{P}(\mathrm{mm})$ & $\mathrm{Ev}(\mathrm{mm})$ & $\mathbf{Q}\left(\mathrm{m}^{3} / \mathrm{s}\right)$ & $\mathbf{Q}(\mathrm{mm})$ & $\mathbf{Q r}(\mathrm{mm})$ \\
\hline Enero & 51,50 & 117,09 & 1,229 & 0,784 & 0,784 \\
Febrero & 57,17 & 118,28 & 2,226 & 1,282 & 1,282 \\
Marzo & 30,05 & 111,18 & 1,587 & 1,012 & 1,012 \\
Abril & 1,15 & 93,39 & 0,838 & 0,517 & 0,517 \\
Mayo & 0,15 & 78,80 & 0,694 & 0,443 & 0,443 \\
Junio & 0,64 & 70,90 & 0,679 & 0,419 & 0,419 \\
Julio & 0,52 & 69,41 & 0,658 & 0,420 & 0,420 \\
Agosto & 0,99 & 69,38 & 0,631 & 0,402 & 0,402 \\
Septiembre & 1,40 & 75,28 & 0,606 & 0,374 & 0,374 \\
Octubre & 1,32 & 91,62 & 0,575 & 0,367 & 0,367 \\
Noviembre & 2,59 & 102,08 & 0,619 & 0,382 & 0,382 \\
Diciembre & 12,44 & 111,79 & 0,614 & 0,392 & 0,392 \\
Suma & 159,91 & 1109,19 & 10,957 & 6,79 & \\
Promedio & 13,33 & 92,43 & 0,913 & 0,57 & \\
Desv. Est. & 21,02 & 19,31 & 0,515 & 0,30 & \\
Máximo & 57,17 & 118,28 & 2,226 & 1,28 & \\
Mínimo & 0,15 & 69,38 & 0,575 & 0,37 & \\
a & $\mathrm{b}(\mathrm{mm})$ & $\mathrm{c}$ & $\mathrm{d}$ & $\mathrm{Sw} \mathrm{w}_{0}(\mathrm{~mm})$ & $\mathrm{Sg} \mathrm{g}_{0}(\mathrm{~mm})$ \\
0,81569 & 345,00000 & 0,21100 & 0,00100 & 22,00000 & 24,36580 \\
\hline & & & & &
\end{tabular}

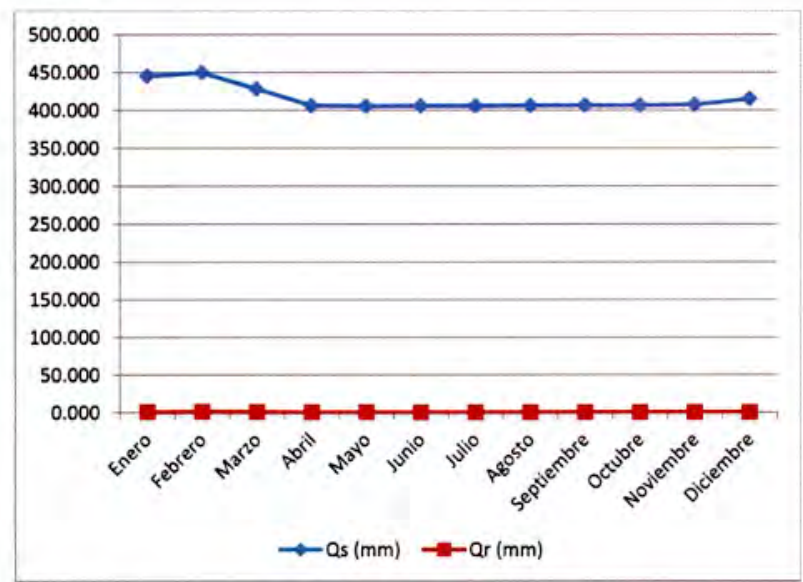

Figura 5. Análisis comparativo caudales simulados y registrados generación 1

metodología empleada, aceptamos que en primera instancia los valores numéricos sean muy discrepantes y con el transcurso del tiempo se espere una convergencia mucho mayor entre los valores observados y simulados.

Asimismo, luego de una serie de simulaciones realizadas, obteniendo en cada proceso 1000 archivos y considerando la extensa disponibilidad de datos en el proceso de calibración, no es recomendable mostrar la gran extensión de data obtenido por lo que procedemos a mostrar los resultados favorables alcanzados en el proceso de calibración de parámetros y mostramos los mejores resultados.

En la Tabla 3 se muestran los mejores valores obtenidos y en la Figura 6 la comparación de caudales simulados y registrados.

Luego de las simulaciones efectuadas, se observa
Tabla 3. Parámetros y simulación usando Generación 1000

\begin{tabular}{llllll}
\hline Mes & $\mathbf{P}(\mathrm{mm})$ & $\mathbf{E v}(\mathrm{mm})$ & $\mathbf{Q}\left(\mathrm{m}^{3} / \mathrm{s}\right)$ & $\mathbf{Q}(\mathrm{mm})$ & $\mathbf{Q r}(\mathrm{mm})$ \\
\hline Enero & 51,50 & 117,09 & 1,229 & 0,784 & 0,784 \\
Febrero & 57,17 & 118,28 & 2,226 & 1,282 & 1,282 \\
Marzo & 30,05 & 111,18 & 1,587 & 1,012 & 1,012 \\
Abril & 1,15 & 93,39 & 0,838 & 0,517 & 0,517 \\
Mayo & 0,15 & 78,80 & 0,694 & 0,443 & 0,443 \\
Junio & 0,64 & 70,90 & 0,679 & 0,419 & 0,419 \\
Julio & 0,52 & 69,41 & 0,658 & 0,420 & 0,420 \\
Agosto & 0,99 & 69,38 & 0,631 & 0,402 & 0,402 \\
Septiembre & 1,40 & 75,28 & 0,606 & 0,374 & 0,374 \\
Octubre & 1,32 & 91,62 & 0,575 & 0,367 & 0,367 \\
Noviembre & 2,59 & 102,08 & 0,619 & 0,382 & 0,382 \\
Diciembre & 12,44 & 111,79 & 0,614 & 0,392 & 0,392 \\
Suma & 159,91 & 1109,19 & 10,957 & 6,79 & \\
Promedio & 13,33 & 92,43 & 0,913 & 0,57 & \\
Desv. Est. & 21,02 & 19,31 & 0,515 & 0,30 & \\
Máximo & 57,17 & 118,28 & 2,226 & 1,28 & \\
Mínimo & 0,15 & 69,38 & 0,575 & 0,37 & \\
a & $\mathrm{b}(\mathrm{mm})$ & $\mathrm{c}$ & $\mathrm{d}$ & $\mathrm{S} \mathrm{w}_{0}(\mathrm{~mm})$ & $\mathrm{Sg} \mathrm{g}_{0}(\mathrm{~mm})$ \\
0,81569 & 299,43250 & 0,17324 & 0,92676 & 428,57140 & 489,23680 \\
\hline
\end{tabular}

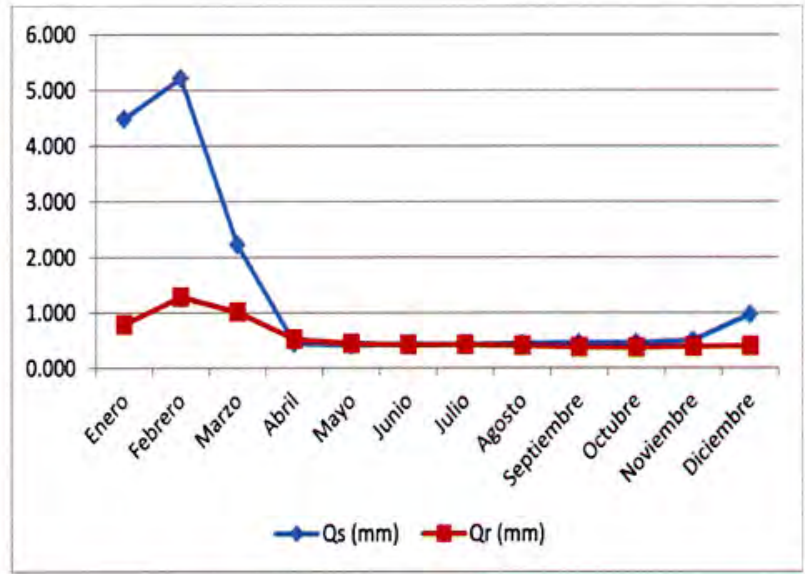

Figura 6. Análisis comparativo caudales simulados y registrados generación 1000

que los valores de descargas observadas son similares a los simulados en periodos de estiaje, situación que nos llama la atención, puesto que este modelo de algoritmos genéticos no permite reproducir descargas en épocas de avenidas.

\section{Análisis estadístico comparativo}

Complementariamente se estableció una correlación entre los caudales registrados y simulados. En la Figura 7 se puede observar dicha correlación lineal con un coeficiente de determinación $\mathrm{r}^{2}$ alto de 0,7835 , lo que representa un buen desempeño de los algoritmos genéticos en la determinación de parámetros del modelo de Thomas y su posterior empleo en el proceso de simulación de caudales. Exceptuando la situación que no tiene buenos resultados en épocas de avenidas o de descargas máximas. 
Pino, E. et al., Uso de algoritmos genéticos para la calibración de un modelo hidrológico precipitación-escorrentia en la cuenca del Caplina

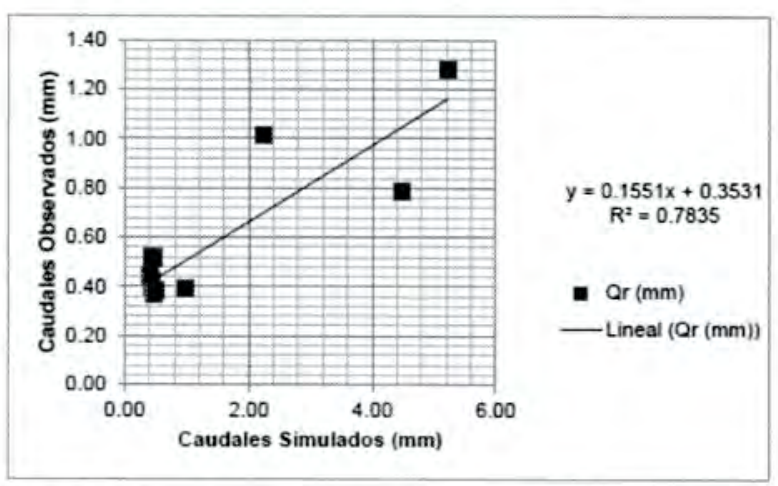

Figura 7. Correlación entre caudales simulados y registrados

\section{CONCLUSIONES}

1. Los algoritmos genéticos poseen características que los hacen altamente robustos para resolver problemas de optimización, toma de decisiones, diseño y otros usos en la ingeniería. Cuando efectuamos la comparación con métodos tradicionales de optimización, y se observa que en general, se sacrifica eficacia para ganar más eficiencia y robustez.

2. El extendido aprovechamiento que se le está dando a los algoritmos genéticos es real. No obstante, para la ingeniería en nuestro país, todavía no se ha explotado esta útil herramienta, como ocurre en muchos países del mundo. En la actualidad aún existe espacio dentro de la teoría de los algoritmos genéticos para continuar con la investigación. No solamente indagando sobre su efectividad sino también extendiendo el abanico de aplicaciones que se le puedan dar.

3. Los resultados obtenidos en las diferentes fases de calibración del modelo aplicado a la cuenca Caplina abren la especulación sobre las posibles fuentes de error. Estas se resumen así: imposibilidad misma de la calibración, la mala adopción de la función objetivo o la existencia de deficiencias en el algoritmo genético. De tratarse el tercer caso, otros operadores o codificaciones deberán intentarse y compararse con el presente trabajo. Se presume, sin embargo, que si el algoritmo genético empleado fuese en realidad deficiente, no se habría dado la posibilidad de calibrar el modelo en la cuenca Caplina.

4. En este trabajo se ha mostrado la evolución del proceso de ajuste de los caudales simulados y observados, asi en la simulación 1 se puede apreciar la gran diferencia que existe entre dichos valores mas no en la simulación 1000 donde se logra un ajuste muy bueno en los meses de estiaje, mas no en los meses de máximas descargas.

5. Las soluciones obtenidas mostraron ser coherentes en similitud a la distribución de caudales observados, con un coeficiente de correlación de 0,7835 . No obstante, para entrar a comparar sobre cuál de las metodologías fue mejor, se requiere analizar otros aspectos tales como los tiempos requeridos para la búsqueda, los computadores empleados, la ausencia o presencia de comandos secundarios que reduzcan la velocidad (como por ejemplo, registro de estadísticas), el número de semillas empleadas, etc.

\section{REFERENCIAS BIBLIOGRÁFICAS}

De Jong, K., (1999), Genetic algorithms: a 30 year perspective. Universidad de Michigan. Festschrift Conference in Honor of John Holland. [Ann Arbor, Michigan, Estados Unidos].

Grefenstette (1993), Hipótesis Estática de los Bloques Constructores.

Goldberg, D., (1989), Genetic algorithms in search, optimization, and machine learning. Reading, Massachusetts, Estados Unidos: Addison-Wesley, 412 p. ISBN 0-201-157675.

Goldberg, D. and Segrest, P., (1987). Finite markov chain analysis of genetic algorithms. Tuscaloosa, Alabama: University of Alabama. En: Proceedings of the Second International Conference on Genetic Algorithms. 1987.p.1-8.

Holland, J., (1992), Adaptation in Natural and Artificial Systems. Cambridge, Massachusetts: MIT Press. Edición 2. 211 p. ISBN 0-262-8213-6.

Karr, C. and Freeman, M., (1999), Industrial applications of genetic algorithms. Boca Ratón, Florida, Estados Unidos: CRC Press. ISBN 0-8493-9801-0.

\section{Correspondencia:}

Edwin Pino Vargas: epino68@hotmail.com

Luis Cornejo Navarrety: lacornejo@hotmail.com

Fecha de Recepción: 22/09/2014

Carmen Román Arce: romancarmen@outlook.com

Fecha de Aceptación: 10/11/2014 